\title{
Nose Whistler Dispersion as a Measure of Magnetosphere Electron Temperature'
}

\author{
H. Guthart \\ Stanford Research Institute, Menlo Park, Calif.
}

(Received May 4, 1965; revised June 21, 1965)

\begin{abstract}
The fractional deviation of nose whistler group delay from the zero-temperature model has been calculated assuming a Maxwellian magnetosphere and a gyrofrequency electron density distribution; i.e., the electron density varies as the inverse cube with distance from the earth's center. The thermal correction to the zero-temperature group refractive index has been inserted into the nose-whistler group delay integral to determine the modified group delay. Significant deviations from the zerotemperature group delay for frequencies above the nose frequency have been calculated. Since deviations from the zero-temperature dispersion of a few percent are readily discernible, nose whistler data should provide at least an estimate of the upper bound on magnetosphere temperature. Twentythree whistlers have been analyzed and an upper bound on magnetosphere temperat ure of $2\left(10^{4}\right)^{\circ} \mathrm{K}$ has been determined.
\end{abstract}

\section{Introduction}

Whistlers are naturally occurring electromagnetic signals in the audio frequency range that result from the dispersion of lightning energy which has traveled through the outer ionosphere along the lines of force of the earth's magnetic field. The term nose whistler designates a particular class of whistlers that exhibits simultaneous rising and falling tones starting at a given frequency called the nose frequency. The dispersion of the energy from the lightning source results from the action of free electrons in the presence of a circularly polarized electromagnetic wave (whose sense of rotation is the same as that of the free electrons in the ambient magnetic field).

${ }^{1}$ This study was sponsored by the Stanford Research Institute. Some assistance was provided by the Air Force Office of Scientific Research on Grant AF-AFOSR-783-65 with Stanford University.
A multipath whistler is a group of whistler traces originating from a single atmospheric that traverse different magnetosphere paths. A typical frequencytime characteristic of a multipath whistler is illustrated by the spectrogram of figure 1 . The spectrogram shows the whistler amplitude, measured by the display intensity, as a function of time (abscissa) and frequency (ordinate). The vertical lines in the spectrogram indicate impulsive atmospherics. The horizontal lines indicate constant-frequency signals, usually power-line harmonics. The three strongest whistler traces of figure 1 are identified at the bottom of the spectrogram by the letters A, B, and C.

Each of the three marked traces is in reality a group of several whistler-components, a fact most easily seen in Trace B. The nose frequency of the leading whistler of each of the three traces is indicated by the frequency indicated to the left of the spectrogram.

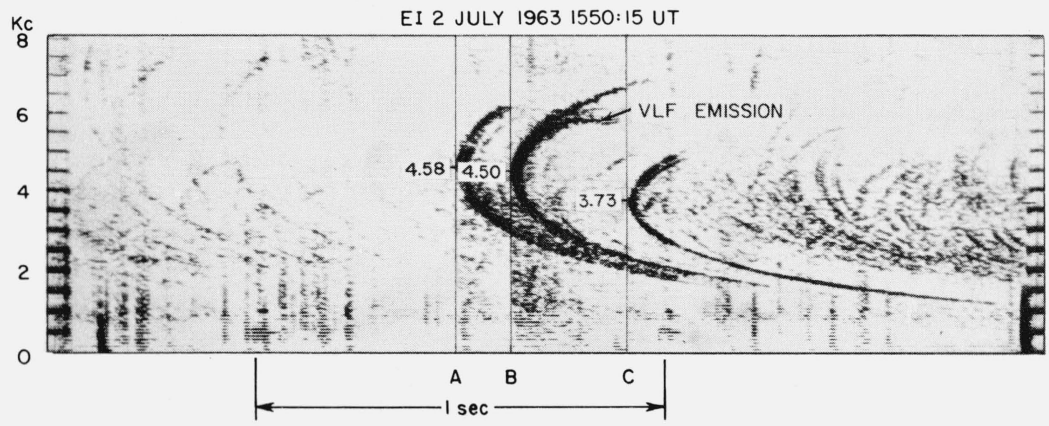

FIGURE 1. Typical nose whistler spectrogram. 
The whistler traces are derived from a single atmospheric source some 1.78 sec prior to the arrival of the nose of Trace A. This source is not shown in the figure, but its character is very much like that of many of the vertical lines seen in figure 1 .

The nose whistler upper cutoff frequency is rarely as much as 60 percent greater than the nose frequency. The upper cutoff frequency of nose whistlers is most often extremely sharp (Trace A and Trace C). The damping at cutoff is of the order of $20 \mathrm{~dB}$ for frequency changes about the cutoff frequency of less than 2 percent. In many instances VLF emissions are triggered at the upper end of nose whistlers (Trace B); in a few cases, the upper cutoff is defined by a more gradual rate of change of attenuation of whistler energy with frequency.

Recently, Scarf [1962] and Liemohn and Scarf [1962a, b] have examined the thermal attenuation of whistlers at the upper cutoff frequency in an effort to estimate the magnetosphere temperature. Assuming isotropic Maxwellian and Cauchy distributions of electron velocity, magnetosphere temperatures of $10^{5}{ }^{\circ} \mathrm{K}$ (Maxwellian) and $4 \times 10^{4}{ }^{\circ} \mathrm{K}$ (Cauchy) were estimated. In a later work, Liemohn and Scarf [1964] note that the mean thermal energy (temperature) is probably lower than they had previously anticipated. In the succeeding sections of this paper, the dispersion characteristics of nose whistlers will be used to set an upper bound on the magnetosphere temperature.

There has been a considerable divergence in magnetosphere temperature estimates. The earliest estimates, based upon the rate of escape of helium [Spitzer, 1948] placed the magnetosphere temperature between 1000 and $2000{ }^{\circ} \mathrm{K}$. Similar temperatures for the lower magnetosphere have been proposed based upon satellite drag calculations [Harris and Jastrow, 1959], incoherent backscatter experiments [VanZandt and Bowles, 1960], and solar Lyman- $\alpha$ line-width measurements [Bates and Patterson, 1961]. In contrast, Chapman [1957] estimates the temperature of the outer magnetosphere at $2 \times 10^{5}{ }^{\circ} \mathrm{K}$. Chapman's estimate is predicated on an estended corona model derived from considering the heat outflow from the sun. Chapman further suggests a temperature gradient of $1{ }^{\circ} \mathrm{K}$ per $\mathrm{km}$ extending from the earth's surface to 5 earth radii.

\section{Theoretical Analysis}

The existence of nose whistlers hus been explained by cold plasma theory; however, the zero-temperature analysis can be modified to include finite particle temperatures. The refractive index $n_{p}$ [Ginzburg, 1961] of the "whistler" mode in a "temperate" plasma is given by

$n_{p}=\left[1+\frac{\frac{1}{2}(a / c)^{2} f_{p}^{2} f}{2\left(f_{H}-f\right)^{3}}\right]\left[\frac{f_{p}}{f^{1 / 2}\left(f_{H}-f\right)^{1 / 2}}\right] \quad\left[1+\frac{1}{2} \frac{f\left(f_{H}-f\right)}{f_{p}^{2}}\right]$.
If the final bracket on the right-hand side of ( 1 ) is approximated by unity, the group refractive index, $n_{g}$, becomes

$$
\begin{aligned}
& n_{g}=\frac{d\left(n_{p} f\right)}{d f}=\frac{\left(\frac{1}{2}\right) f_{p} f_{H}}{f^{1 / 2}\left(f_{H}-f\right)^{3 / 2}} \\
& +\frac{\left(\frac{1}{2}\right)(a / c)^{2} f_{p}^{3} f^{1 / 2}\left[(3 / 2) f_{H}+2 f\right]}{2\left(f_{H}-f\right)^{9 / 2}}, \\
& \text { for } \frac{f\left(f_{H}-f\right)}{f_{p}^{2}}<<\frac{\left(\frac{1}{2}\right)(a / c)^{2} f_{p}^{2} f}{\left(f_{H}-f\right)^{3}}<<1,
\end{aligned}
$$

and where

$f=$ wave frequency,

$f_{p}=$ plasma frequency,

$f_{H}=$ electron gyrofrequency,

$a=\left[\frac{2 k T}{m}\right]^{1 / 2}=$ root mean square electron speed,

$T=$ Electron temperature,

$k=$ Boltzmann's constant,

$m=$ electron mass,

$c=$ speed of light.

The whistler time delay, $t$, can be determined at each frequency by an integration of the group refractive index over the whistler path:

$$
t=\int_{S} \frac{n_{g} d s}{c}
$$

where $d s$ is the elemental path length and the propagation is along the magnetic field. The whistler dispersion, $D$, is defined by:

$$
D=t f^{1 / 2}=D_{0}+D_{1}
$$

where

$$
\begin{aligned}
& D_{0}=\frac{1}{2 c} \int_{S} \frac{f_{p} f_{H} d s}{\left(f_{H}-f\right)^{3 / 2}}, \\
& D_{1}=\frac{1}{2 c} \int_{S}\left(\frac{1}{2}\right)(a / c)^{2} \frac{f_{p}^{3} f\left[(3 / 2) f_{H}+2 f\right]}{\left(f_{H}-f\right)^{9 / 2}} d s .
\end{aligned}
$$

Here $D_{0}$ defines the cold plasma dispersion, and $D_{1}$ the finite temperature perturbation to the zerotemperature analysis. 
The integrals may be evaluated subject to some assumptions on the magnetospheric whistler environnent and some changes of variable. Assume that: 1) the earth is immersed in a dipole magnetic field; 2) the magnetosphere has isothermal Maxwellian parwcle distribution; and (3) the magnetosphere electron density is proportional to the electron gyrofrequency, so that $f_{p} \propto f_{H}^{1 / 2}$. The final assumption is consistent with the observations of Smith [1961] based upon whistler dispersion measurements below the nose frequency.

Express the path length, $S$, in terms of the variable $x$, where $x$ is the sine of the magnetic latitude, $\theta$. If $x$ is subsequently expressed in terms of the quantity $y$, where $y$ is defined by

$$
1-y^{2}=\frac{\left(1-x^{2}\right)^{3}}{\left(1+3 x^{2}\right)^{1 / 2}}=\frac{f_{H 1}}{f}
$$

then the dispersion of a whistler propagating along a magnetic field line can be expressed by

$$
D_{0}=\frac{r_{0}}{c f_{H 0}^{1 / 2} \cos ^{5} \theta_{0}} \int_{0}^{y_{0}} \frac{f_{p} h(y) d y}{\left(1-m+m y^{2}\right)^{3 / 2}},
$$

and

$$
D_{1}=D_{1}^{\prime}+D_{1}^{\prime \prime}
$$

where

$$
\begin{aligned}
D_{1}^{\prime} & =\frac{3 r_{0} f(a / c)^{2}}{4 c f_{H 0}^{7 / 2} \cos ^{23} \theta_{0}} \int_{0}^{y_{0}} \frac{f_{p}^{3} h(y)\left(1-y^{2}\right)^{3} d y}{\left(1-m+m y^{2}\right)^{9 / 2}}, \\
D_{1}^{\prime \prime} & =\frac{r_{0} f^{2}(a / c)^{2}}{c f_{H 0}^{9 / 2} \cos ^{29} \theta_{0}} \int_{0}^{y_{0}} \frac{f_{p}^{3} h(y)\left(1-y^{2}\right)^{4} d y}{\left(1-m+m y^{2}\right)^{9 / 2}}, \\
h(y) & =\frac{2}{9} \frac{\left(1+3 x^{2}\right)^{5 / 3} y}{\left[1+(5 / 3) x^{2}\right]\left[1-y^{2}\right]^{1 / 6} x} .
\end{aligned}
$$

Here $r_{0}$ is the radius of the earth, $\theta_{0}$ is the latitude of the termination of the field line along the earth's surface, $f_{H 0}$ is the gyrofrequency at the equator on the earth's surface, and $m$ is the wave frequency, $f$, normalized to the minimum gyrofrequency along the path, $f_{H 1}$.

The symbols $y$ and $h(y)$ are plotted in figure 2 . For most latitudes of interest, $y_{0}$ is very close to unity (for $\theta_{0}>55^{\circ}, y>0.99$ ); consequently we shall assume $y_{0}=1$. The function $h(y)$, too, is well behaved despite the singularity at $y=1$. To evaluate the integrals of (5) and (6), we shall remove the factor $h(y)$ from the integrals. The error introduced by these assumptions shall be considered below.

The magnetosphere electron density is now assumed proportional to the electron gyrofrequency, so that

$$
f_{p}=9 C^{1 / 2} f_{H}^{1 / 2}=9 C^{1 / 2} f_{H}^{1 / 2}\left(1-y^{2}\right)^{-1 / 2},
$$

where $C$ is the constant of proportionality between density and gyrofrequency and is approximately $2\left(10^{4}\right)$ when the electron density is $250 \mathrm{~cm}^{-3}$ at 4 earth radii.

The integrals of (5) and (6) can now be represented [T. F. Bell, private communication; Guthart, 1964]

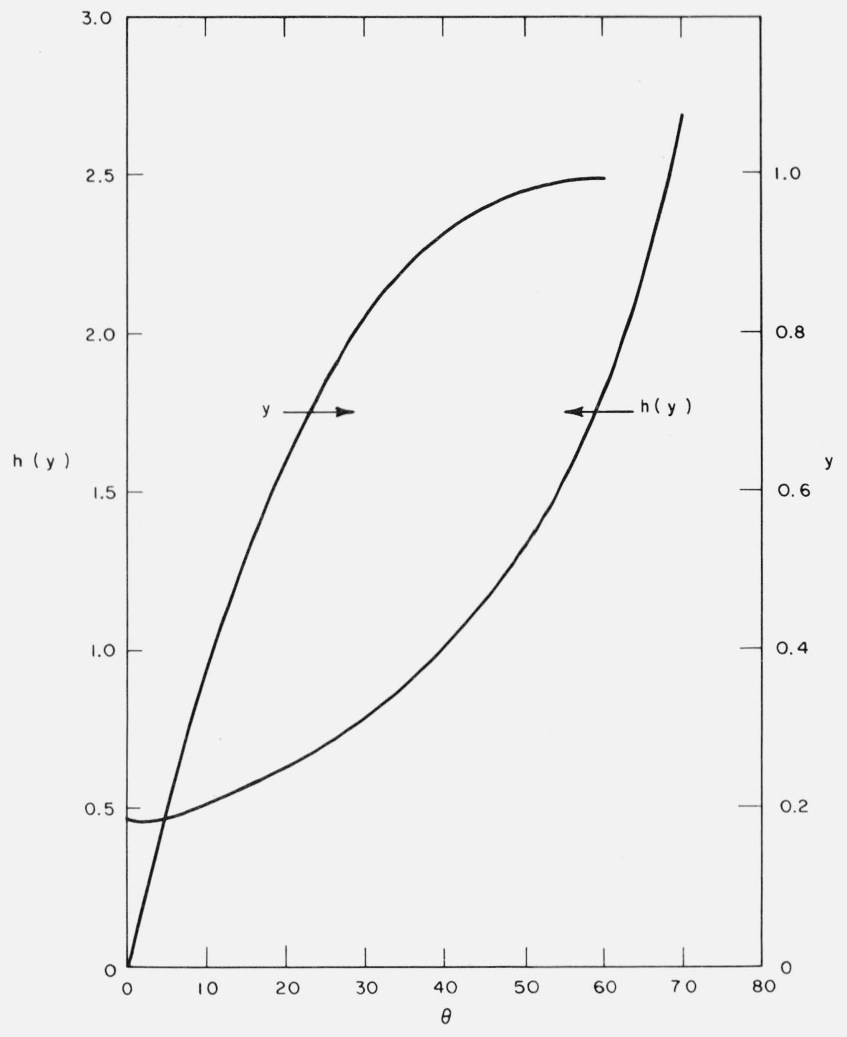

FIGURE 2. Quantities y and $\mathrm{h}(\mathrm{y})$ as a function of magnetic latitude, $\theta$.

in terms of elliptic functions as shown below:

$$
\begin{gathered}
I_{0}=\int_{0}^{\prime} \frac{d y}{\left(1-y^{2}\right)^{1 / 2}\left(1-m+m y^{2}\right)^{3 / 2}}=\frac{E(m)}{(1-m)}, \\
I_{1}^{\prime}=\int_{0}^{\prime} \frac{d y\left(1-y^{2}\right)^{3 / 2}}{\left(1-m+m y^{2}\right)^{9 / 2}}=\frac{4}{105} \frac{d^{2}}{d m^{2}} \\
{\left[\frac{-K(m)}{(1-m)}+\frac{2(2-m)}{(1-m)^{2}} E(m)\right],}
\end{gathered}
$$

and

$$
I_{1}^{\prime \prime}=\int_{0}^{\prime} \frac{d y\left(1-y^{2}\right)^{5 / 2}}{\left(1-m+m y^{2}\right)^{9 / 2}}=\frac{8}{105} \frac{d^{3}}{d m^{3}} \frac{E(m)}{1-m},
$$

where $E$ is the complete elliptic integral of the second kind and $K$ is the elliptic quarter period [MilneThompson, 1950].

The normalized first-order disperson, $\left(D_{1} / D_{0}\right)$, is expressible in terms of these elliptic functions and is given by

$$
\frac{D_{1}}{D_{0}}=\frac{3(a / c)^{2}(81 C) F(m)}{4 f_{H 1}},
$$


where

$$
F(m)=\frac{m(1-m)\left[I_{1}^{\prime}(m)+\left(\frac{4}{3}\right) m I_{1}^{\prime \prime}(m)\right]}{E(m)} .
$$

Here $\boldsymbol{F}(m)$ is defined by the solutions of the elliptic integrals which have been evaluated using the wellknown differential relations between the functions; $F(m)$ is shown in figure 3 for $0.25 \leqslant m \leqslant 0.75$. From the figure it is seen that $F(m)$ is a rapidly varying function of normalized frequency, $m$.

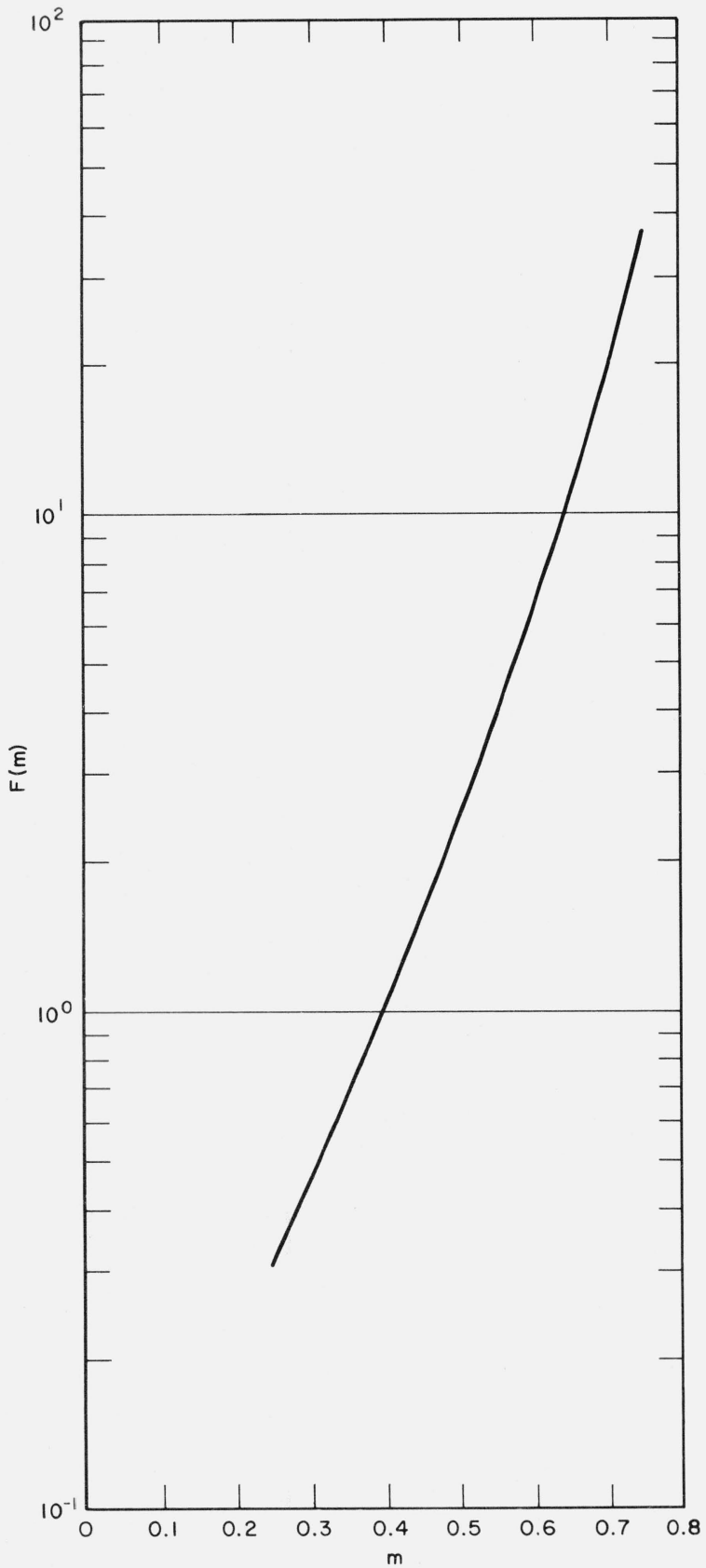

FigURE 3. Elliptical integral weighting factor, $\mathrm{F}(\mathrm{m})$, as a function of normalized frequency.
Equation (10) can now be used to measure the magnetosphere temperature. Nose whistler data can be analyzed to determine whether there is a measurable increase in dispersion above the zero-temperature predictions. If there is a measurable increase in dispersion, then (10) measures the temperature directly. If no measurable increase is observed, (10) sets an upper bound on the temperature of the magnetosphere, depending upon the accuracy to which the dispersion can be measured.

The integrals of (7) through (9) have been evaluated, without approximations for $y_{0}$ and $h(y)$, when $m=0.55$ and $m=0.65$. The resulting solutions have been expressed in the form $\left(D_{1} / D_{0}\right)$ and compared to the approximate solution of (10). The exact integral solution is then seen to yield a value of $F(m)$ that is 80 percent of the value determined from the elliptic function approximation.

A further examination of the integrands of the firstorder dispersion integrals leads to the conclusion that at least 80 percent $(m \geqslant 0.55)$ of the increased dispersion introduced by temperature occurs within $10 \mathrm{deg}$ $(y=0.352)$ of the geomagnetic equator. This justifies the statement that an isothermal magnetosphere is unnecessary and (10) measures the electron temperature at the radial extremum of the magnetic field line.

\section{Dispersion Analysis of Nose Whistler Data}

When more than one nose whistler appears in a multipath whistler, the nose frequency of the succeeding nose whistlers almost always decreases in time, which is consistent with the observation that whistlers with lower nose frequencies traverse higher frequency field lines, and hence undergo more delay. The fact that nose whistlers sometimes appear out of succession in a whistler group has led to the deduction by Carpenter [1963] of a knee in the magnetospheric ionization density profile.

The nose whistlers used in this analysis were accumulated by Stanford University at Eights Station (EI), Antarctica, Byrd Station (BY), Antarctica, and Suffield Experimental Station (SES), Alberta. The techniques for recording and spectrographic data display are described in a Stanford Radioscience Laboratory report [Carpenter, 1960]. A discussion of the detailed procedure for the evaluation of the spectrographic data leading to magnetosphere temperature estimates is found in Guthart [1964]. Briefly, the procedure for data analysis is to scale the nose whistler spectrogram to determine:

(1) The nose frequency, $f_{n}$,

(2) the upper cutoff frequency, $f_{m}$,

(3) the travel time to the nose from the whistler source, $t_{n}$,

(4) the travel time to the upper cutoff frequency from the whistler source, $t_{m}$.

The measured travel time at the upper cutoff frequency can be normalized to the travel time at the nose 
frequency $\left(t_{m} / t_{n}\right)$. The ratio can now be compared to the computed zero-temperature, dispersion curves of J. Angerami [private communication].

Angerami computed dispersion curves for an electron density model given by

$$
N=\text { electron density }=C_{1}\left(r_{0} / r\right)^{3} .
$$

In contrast, (10) is evaluated for a gyrofrequency electron density model; i.e.,

$$
N=C f_{H}=C_{2}\left(r_{0} / r\right)^{3}\left(1+3 \sin ^{2} \theta\right)^{1 / 2} .
$$

where $r_{0}$ is the radius of the earth, $r$ is the radial distance from the center of the earth, and $\theta$ is the magnetic latitude. Computations of normalized dispersion using these two models for electron density yield results differing by less than 1 percent $(m<0.7)$, a negligible error. More generally, the normalized dispersion is not critically dependent on the assumption that the magnetosphere electron density varies as $\left(r_{0} / r\right)^{3}$. If the magnetosphere electron density were, in fact, to vary as $\left(r_{0} / r\right)^{2}$ or $\left(r_{0} / r\right)^{4}$, then, for the whistlers accumulated in this study (these whistlers shall be discussed shortly), the normalized dispersion would change by less than 2 percent from the $\left(r_{0} / r\right)^{3}$ value.

The comparison of the experimental and theoretical quantities results in a value for the increase in dispersion introduced by the magnetospheric electron temperature; i.e., the comparison yields the quantity $D_{1} / D_{0}$, on the left-hand side of (10). The minimum value of $D_{1} / D_{0}$ is specified by the scaling accuracy, and is substituted into (10) when the experimental and theoretical travel times are in agreement to the limit of the experimental accuracy.

The scaled nose frequency and the travel time to the nose frequency are used in conjunction with the zerotemperature dispersion curves of Angerami to determine the minimum gyrofrequency along the path and the constant of proportionality, $C$, relating the electron density and the electron gyrofrequency. The change in nose frequency introduced by a finite electron temperature is negligible for some typical nose whistler parameters [Guthart, 1964].

Equation (10) can now be solved for temperature. If a deviation in dispersion is observed, the inferred temperature is the electron temperature of the magnetosphere. If no additional dispersion is observed, then we take the minimum observable dispersion from which we derive an upper bound on the magnetosphere electron temperature, since a higher temperature would have generated an observable dispersion.

The accuracy of our estimate of magnetosphere temperature is critically dependent on the accuracy of our measurement of dispersion of the upper cutoff frequency. In reality, the measured quantity is the normalized dispersion, $D_{m} / D_{n}$, i.e., the dispersion at the upper cutoff frequency normalized to the dispersion at the nose frequency. Thus, $D_{m} / D_{n}$ is given by

$$
\frac{D_{m}}{D_{n}}=\frac{t_{m}}{t_{n}}\left(\frac{f_{m}}{f_{n}}\right)^{1 / 2}
$$

The greatest uncertainty in scaling dispersion is in the determination of the time of occurrence of the atmospheric from which the whistler originated, i.e., in determining $t_{n}$. However, in the measurement of normalized dispersion, the desired quantity is $t_{m} / t_{n}$, where

$$
\frac{t_{m}}{t_{n}}=\frac{t_{n}+\triangle t_{m}}{t_{n}}
$$

When $m_{m}=0.65, \triangle t_{m}$ is only 15 percent of $t_{n}$; consequently a 5-percent error in determining the travel time to the nose frequency, $t_{n}$, generates an error of less than 1 percent in the scaling of $\left(t_{m} / t_{n}\right)$. A 5percent determination of $t_{n}$ is readily achieved.

The most common method of atmospheric (sferic) determination is based on the similarity of the traces of whistlers during a given 2-min period (at a given receiving site, the VLF recorder is usually turned on for $2 \mathrm{~min}$ of every hour), or sometimes over a period of many hours. Spectrograms of different whistlers are lined up so that the corresponding whistler traces are superimposed. The causative atmospherics correspond on the superimposed spectrograms. An alternative method is to scale the normalized dispersion of the frequencies below the nose frequency and use the dispersion curves of Angerami to infer the sferic location. The application of this technique to several whistlers within a whistler group permits positive identification of the causative sferic. In this study, both the overlay and scaling techniques were applied so that the travel time to the nose could be determined to better than 3 percent. Carpenter [1960] describes several empirical methods of identifying whistler sources.

The whistler nose frequency can be scaled to within 3 percent and the upper cutoff frequency can be scaled to better than 1 percent. As a consequence of these scaling accuracies, it is estimated that the normalized dispersion, $D_{m} / D_{n}$, can be scaled to within 2 percent. The minimum observable value for $D_{1} / D_{0}$, the quantity that specified the first-order change in dispersion introduced by finite electron temperature, is thus taken to be 0.02 .

Some 23 nose whistlers have been scaled and in no case has the measured dispersion deviated from that predicted by the cold plasma (electron temperature $=0$ ) analysis; i.e., no temperature effects were observed. The whistlers used in this study are listed in table 1, including their respective properties. These whistlers have the following spectrum of parameters:

(1) $0.57 \leqslant m_{m} \leqslant 0.75$

(2) $2.65 \mathrm{kc} / \mathrm{s} \leqslant f_{H 1} \leqslant 13.3 \mathrm{kc} / \mathrm{s} ; 6.9 \geqslant r / r_{0} \geqslant 4$;

(3) $5.9\left(10^{3}\right) \leqslant C \leqslant 35.8\left(10^{3}\right)$;

where $m_{m}=f_{m} / f_{H 1}$ is the upper cutoff frequency normalized to the minimum gyrofrequency along the path, $r / r_{0}$ is the normalized distance from the earth's center, and $C$ is the constant of proportionality relating the electron density and ambient magnetic field 
strength in the gyrofrequency model for electron density. The scaled whistlers include samples from the local summer and winter as well as over the 24-hr period. The data repeatability is such that within a 2-min recording period, numerous whistlers generate similar temperature estimates, e.g., numbers 1 to 3 , table 1. Indeed, over a period of hours much of the nose whistler data will be repeated, e.g., numbers 1 and 6 , table 1 . Further, it should be noted that the data constituting this sample have been selected to demonstrate the existence of enhanced dispersion introduced by finite electron temperatures. Whis- tlers having an upper cutoff frequency below the nose frequency have not been included because, for a given magnetosphere temperature, these whistlers would give rise to less enhancement of the dispersion.

The minimum observed upper bound on magnetosphere electron temperature is $2\left(10^{4}\right){ }^{\circ} \mathrm{K}$ (number 16 , table 1). (For the whistlers of table 1,

$$
\frac{\left(\frac{1}{2}\right)(a / c)^{2} f_{p}^{2} f}{\left(f_{H}-f\right)^{3}} \geqslant 4.5 \frac{f\left(f_{H}-f\right)}{f_{p}^{2}}
$$

TABLE 1. Experimental nose whistler data

\begin{tabular}{|c|c|c|c|c|c|c|c|c|}
\hline & Date & $\begin{array}{l}\text { Time(UT) hr:min/ } \\
\text { sec into run }\end{array}$ & Sta. ${ }^{a}$ & $f_{H 1}$ & $m_{m}=\frac{f_{m}}{f_{H 1}}$ & $C$ & $T^{b}$ & $\frac{\left(t_{m} / t_{n}\right) \exp }{\left(t_{m} / t_{n}\right)_{t h}}$ \\
\hline
\end{tabular}

"Stations EI, Eights, Antaretica; BY, Byrd, Antarctica; ST, Stanford, Calif.

${ }^{b} \mathrm{~T}$ is an upper bound on temperature.

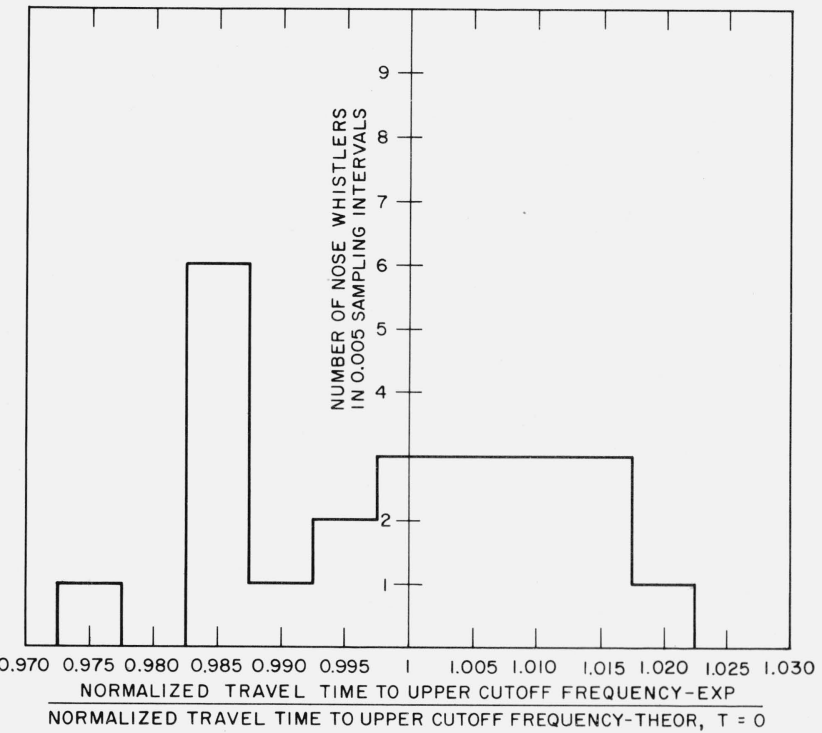

FiguRE 4. Error histogram for travel time to the upper cutoff frequency of nose whistlers.
As a consequence, (2) is approximately valid.) Figure 4 is a histogram depicting the observed error in the normalized travel time to the upper cutoff frequency. The number of nose whistlers exceeding the zerotemperature prediction was 10 ; the number whose time was less than that predicted by the zero temperature theory was 11 . Only in one case was the difference in observed and predicted times greater than 2 percent. As a consequence, a statistical analysis of a larger sample should improve the temperature estimate considerably.

Some of the spectrograms studied in this analysis are shown in figure 5 . The number identifying each whistler corresponds to the similarly numbered whistler of table 1. In analyzing the whistlers selected for temperature evaluation, care had to be exercised to exclude from the analysis VLF emissions triggered at the end of a whistler. (As an example, see number 15 of fig. 5c.) The emission at the end of a whistler was nearly always characterized on the spectrogram by a change in slope (for example, see fig. 5e). However, in some cases, there was sufficient doubt as to the point of origin of the VLF emissions that the whistler trace had to be discarded (for example, see the upper branch of Trace B of fig. 1). 


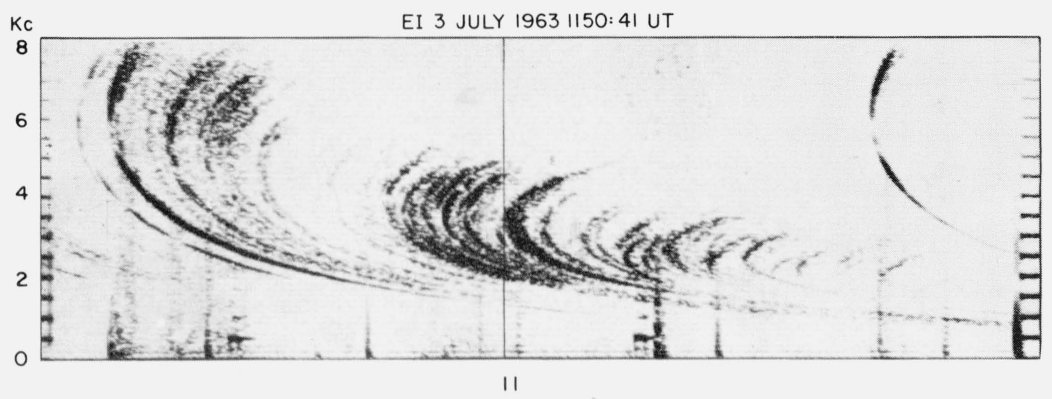

(a)

FIGURE 5a. Spectrograms of nose whistlers used in this analysis.

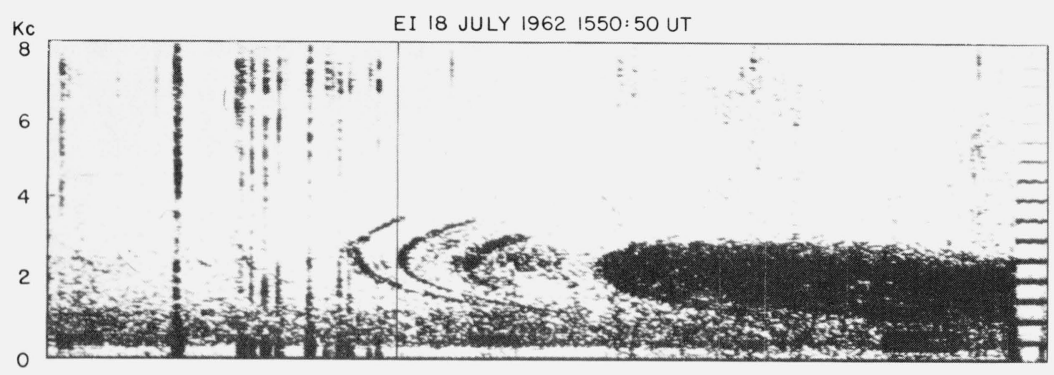

(b)

FigURE 5b. Spectrograms of nose whistlers used in this analvsis.

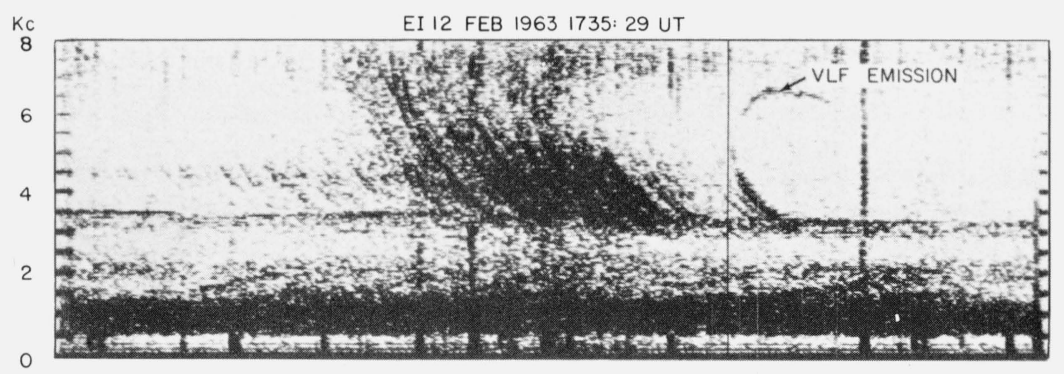

15

(c)

FigURE 5c. Spectrograms of nose whistlers used in this analysis.

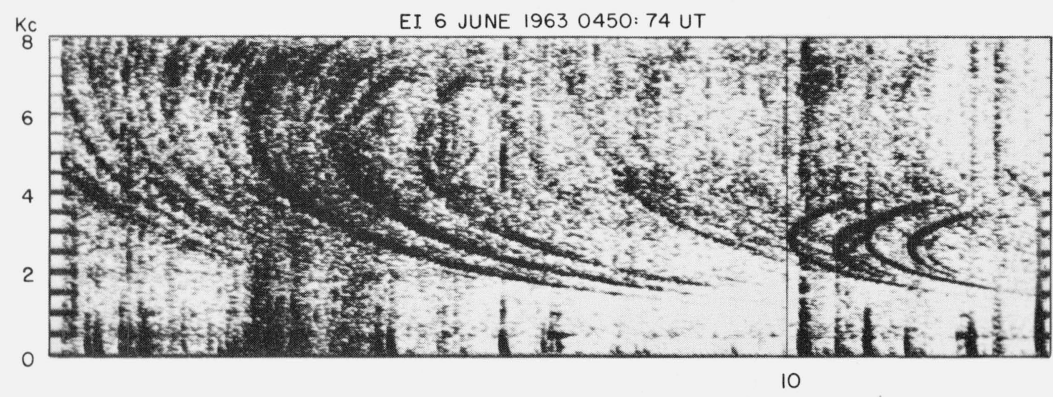

(d)

Figure 5d. Spectrograms of nose whistlers used in this analysis. 


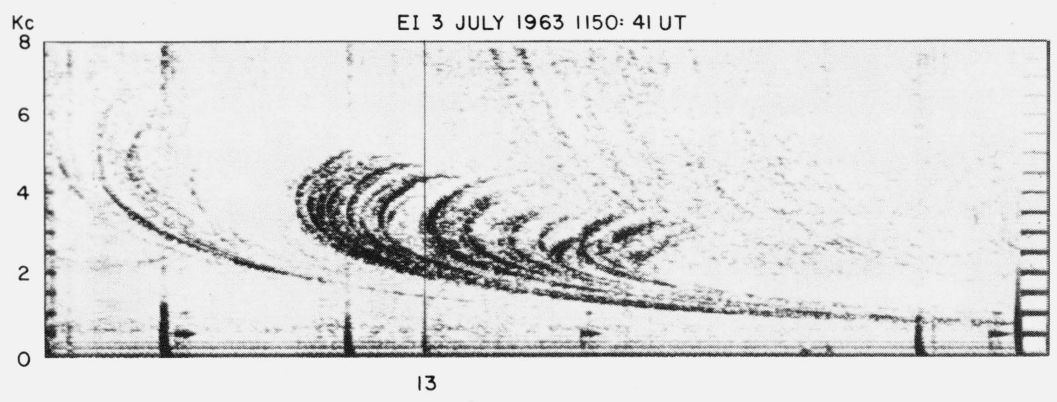

(e)

FigurE 5e. Spectrograms of nose whistlers used in this analysis.

\section{Concluding Remarks}

The increased dispersion introduced by finite electron temperatures has been calculated. The results have been compared to experimentally observed whistlers, and the observed whistlers are seen to exhibit no temperature effects. As a consequence, an upper bound on the magnetosphere electron temperature has been set at $2\left(10^{4}\right){ }^{\circ} \mathrm{K}$, an order of magnitude below the previous bound set by Chapman. With the accumulation of more data samples and the application of statistical analysis, it should be possible to lower the above upper bound on temperature.

The author deeply appreciates the guidance, insight, and stimulating counsel of R. A. Helliwell of Stanford University during the course of the work. The author wishes to thank D. L. Carpenter of Stanford University for his assistance in data accumulation and evaluation and T. F. Bell and R. L. Smith of Stanford University for their general interest and assistance in some mathematical derivations. The author acknowledges with thanks the helpful corrections by H. B. Liemohn of the Southwest Center for Advanced Studies, to an earlier report [Guthart, 1964].

\section{References}

Bates, D. R., and T. N. L. Patterson (1961), An estimate of the temperature in the exosphere, Planetary Space Sci. 5, 328.

Carpenter, D. L. (1960), Identification of whistler sources on visual records and a method of routine whistler analysis, Tech. Rept. 5, Contract AF 18(603)-126 AFOSR-TN-60 315, Stanford Univ., Stanford, Calif.

Carpenter, D. L. (1963), Whistler evidence of a "knee" in the magnetosphere ionization density profile, J. Geophys. Res. 68, $1675-1682$.

Chapman, S. (1957), Notes on the solar corona and the terrestrial ionosphere, Smithsonian Contributions to Astrophysics 2, No. 1, $1-12$.

Ginzburg, V. L. (1961), Propagation of Electromagnetic Waves in Plasma (Gordon and Breach, Science Publishers, Inc., New York, N.Y.).

Guthart, H. (1964), Whistlers in a thermal magnetosphere, Report, Stanford Res. Inst., Menlo Park, Calif.

Harris, I., and R. Jastrow (1959), An interim atmosphere derived from rocket and satellite data, Planetary Space Sci. 1, No. 1, $20-26$.

Liemohn, H. B., and F. L. Scarf (1962a), Exospheric electron temperatures from nose whistler attenuation, J. Geophys. Res. 67, $1785-1789$.

Liemohn, H. B., and F. L. Scarf (1962b), Whistler attenuation by electrons with an $E^{-2.5}$ distribution, J. Geophys. Res. 67, 41634167.

Liemohn, H. B., and F. L. Scarf (1964), Whistler determination of electron energy and density distribution in the magnetosphere, J. Geophys. Res. 69, 883-904.

Milne-Thompson, L. M. (1950), Jacobian Elliptic Function Tables (Dover Publications, New York, N.Y.).

Scarf, F. L. (1962), Landau damping and the attenuation of whistlers, Phys. Fluids 5, 6-13.

Smith, R. L. (1961), Properties of the outer ionosphere deduced from nose whistlers, J. Geophys. Res. 66, 3709-3716.

Spitzer, L. (1948), The Terrestrial Atmosphere Above 300 km, Atmospheres and Planets, ed. C. P. Keuper, pp. 213-249 (Chicago Univ. Press, Chicago, Ill.).

VanZandt, T. E., and K. L. Bowles (1960), The temperature of the $F$-region, abstract, Symposium of the Exosphere and $F$-Region Electron Density Program, Joint Meeting of URSI and IRE. 